\title{
Design of Intelligent Anti-Theft System for Electric Bicycles
}

\author{
Wang Yanan $^{1, a}$, Kang Caiqin ${ }^{2, b}$ \\ ${ }^{1}$ Institute of Electronic and Control Engineering Chang'an University Xi'an City, China \\ ${ }^{2}$ Institute of Information Engineering Chang'an University Xi'an City, China \\ a1603338525@qq.com, b1490546139@qq.com
}

Keywords: microcontroller; electric bicycles; anti-theft; battery; Proteus

\begin{abstract}
According to the shortages of anti-theft methods for electric bicycles, combined with modern electronic technology, single-chip technology and intelligent sensor technology, propose a design based on the microcontroller used for electric bicycles' anti-theft. In this paper the hardware and software of the intelligent anti-theft system are described , which can not only much more conveniently and safely lock or unlock the electric bicycles, but also can timely monitor whether the electric bicycle's battery is stolen, and the simulation results based on the software Proteus are given. The intelligent anti-theft system for electric bicycles is a novel design, which has good security and operability and meet the requirements in the aspect of electric bicycles' anti-theft.
\end{abstract}

\section{Introduction}

At present, electric bicycles become a means of transport because of their advantages such as fast,light,and economical. The development of electric bicycles in Japan, Germany and other European countries is earlier than the others all over the word, and in recent years the United States' Electric bicycle market is rapidly developing[1]. Generally speaking, the global potential market of electric bicycle is huge. Meanwhile in our country a large number of enterprises and institutes do research about it. So electric bicycles play an increasingly important role in people's daily life.

However, theft cases occur frequently, causing great losses to users. People are mainly concentrated on the purchase of the chain,U-lock or place the front lock against theft. But the first two methods are not the parts of the bicycle body, and it's inconvenient to operate,especially in the cramped parking lot or in the rain. As for the front lock, which is the part of the bicycle body though, there are also some shortcomings. The key hole is not easy to find at night. In recent years as the electric bicycle battery prices soar due to the rise in lead price, some thieves aim at batteries which are easier to steal. Battery theft cases often occur, causing trouble to the users.

Therefore, according to the shortages of anti-theft methods for electric bicycles it's necessary and meaningful to design the intelligent anti-theft system for electric bicycles based on the microcontroller.

\section{Design and implementation of the system}

With the rapidly development of our economy and the improvement of people's living standards, the demand for electric bicycles on the market is growing and the competition of all kinds of brands become increasingly intense. The safety, comfortableness, operating convenience have become the important factors when users choose electric bicycles. At present the electric bicycles' anti-theft methods are inconvenient to operate and have low security. Design and realize the intelligent anti-theft system for electric bicycles based on the microcontroller in the paper.

The system's overall design thought. The main purpose is to design and implement the intelligent anti-theft system based on the microcontroller, which can not only much more conveniently and safely lock or unlock the electric bicycle,but also can timely monitor whether the battery is stolen. The intelligent anti-theft system for electric bicycles based on the microcontroller is mainly composed of the password-input module,memory module,power module,battery voltage detection module,alarm module and lock or unlock module.After the user enter the 6-digit password, 
the microcontroller will judge whether the password is correct. If correct, the system will lock or unlock the electric bicycle in response to the indication from the user. At a certain time, the microcontroller will read the pressure value of the electric bicycle' s battery measured by the pressure sensor to monitor whether the battery is stolen. It' s overall system structure is shown in Fig. 1.

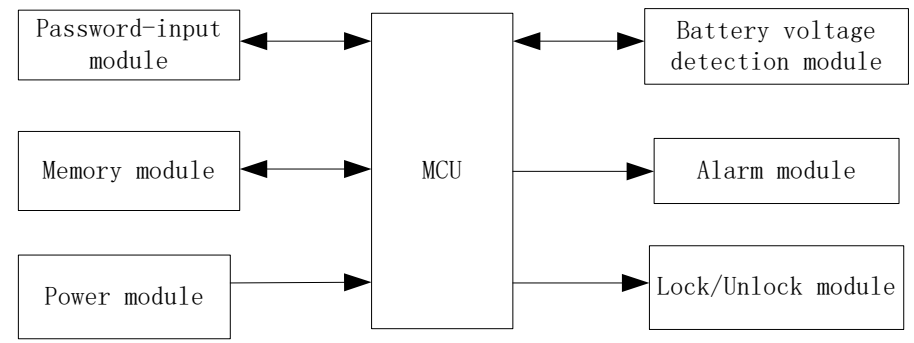

Fig. 1 The overall system structure

Hardware design. The overall hardware schematic diagram of the intelligent anti-theft system electric bicycles based on the microcontroller is shown in Fig. 2, which includes the microcontroller, the matrix keyboard, EEPROM, the power supply module, LCD display, the pressure detection module, the alarm module and the unlock or lock module.

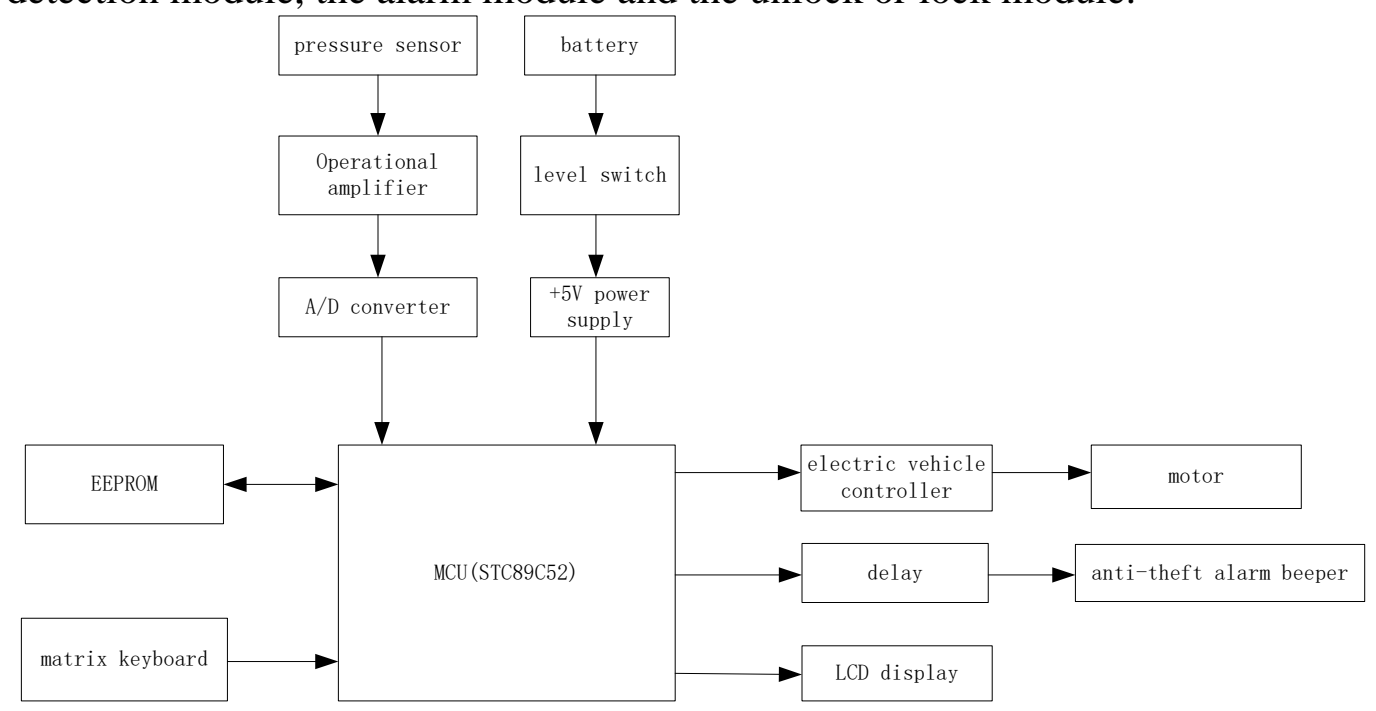

Fig. 2 The overall hardware schematic diagram

In the design the selection of the hardware are as follows:

(1) The STC89C52 microcontroller is chosen which is a kind of low-power,high performance 8-bit CMOS microcontroller and has $8 \mathrm{~K}$ system programmable flash memory.

(2) The AT24C01 memory chip is chosen which is belong to the EEPROM with bus interface and used to store the password.

(3) The LCD12864 display is chosen which can show Chinese characters,numbers and characters.

(4) The strain type pressure sensor is chosen which use elastic and sensitive elements and the strain gauge to convert the measured pressure value into the corresponding change in resistance.

(5) The ADC0809 chip is chosen which is a monolithic CMOS device with an 8-bit analog-to-digital converter,8-channel multiplexer and microprocessor compatible control logic. The 8-bit A/D converter uses successive approximation as the conversion technique.

(6) The regulator chip LM2575 is chosen to convert the voltage from DC $12 \mathrm{~V}$ to DC 5V. Currently the electric bicycle battery is comprised of several DC $12 \mathrm{~V}$ batteries in series on the market. The DC $12 \mathrm{~V}$ battery is converted to DC $5 \mathrm{~V}$ to supply the microcontroller with power.

The system uses STC89C52 microcontroller as the core, the $4 * 3$ matrix keyboard to enter the password , the LCD12864 to show the program running process and the electric bicycle controller to control motor. The pressure measured by the pressure sensor will be sent to the microcontroller through the amplifier and the A/D converter. 
Software design .The overall software flow chart of the anti-theft system electric bicycles based on the microcontroller is shown in Fig. 3, which includes the main program module, the initialization module, the button module, the memory module, the unlock or lock module, the changing password module, the display module and the comparing pressure module.

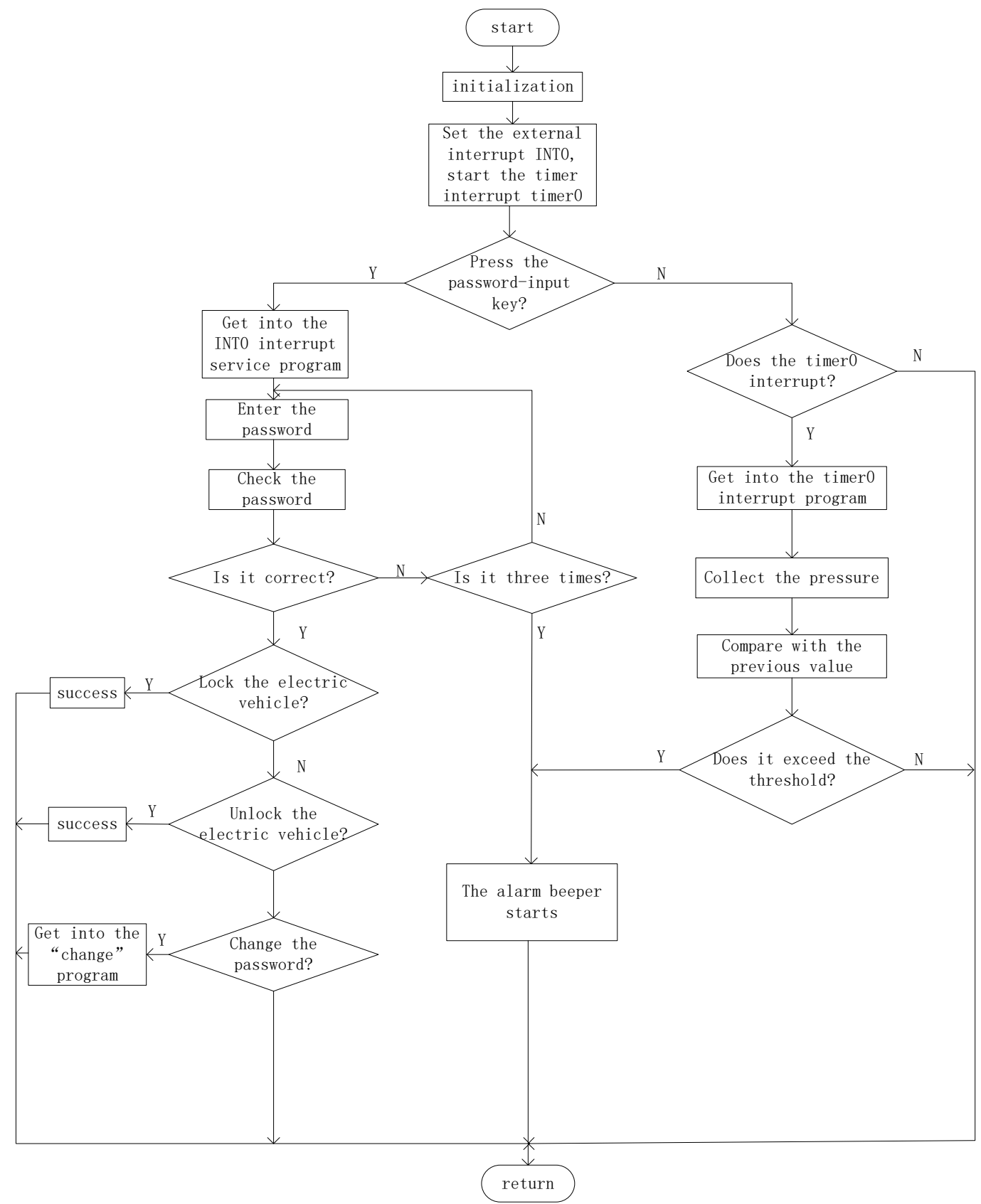

Fig. 3 The overall software flow chart

1) The Button Module. The system uses $4 * 3$ matrix keyboard to enter password and the key subroutine flow chart is shown in Fig. 4. 


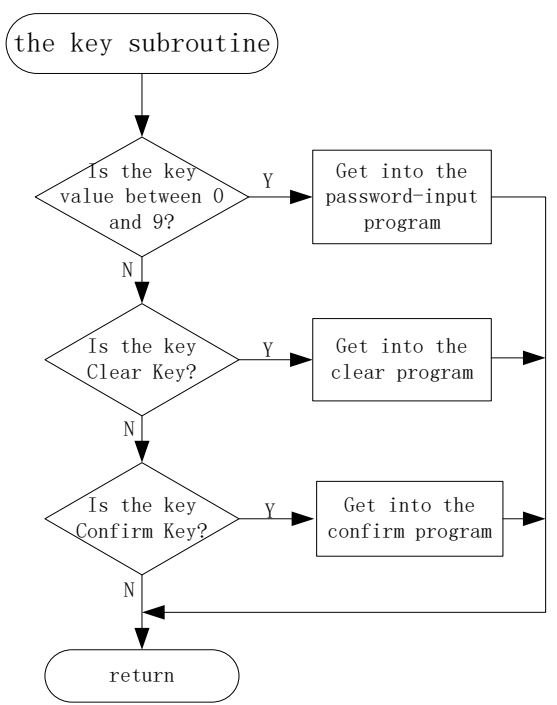

Fig. 4 The key subroutine flow chart

2) The Changing Password Module. When the password is correct the user can select to press the key 3 to set new password, which will be sent to the AT24C01 to be stored. Press the confirm key after the new 6-bit password are all entered and the system will automatically jump to the beginning of the program, and call the new password.The changing password subroutine flow chart is shown in Fig. 5.

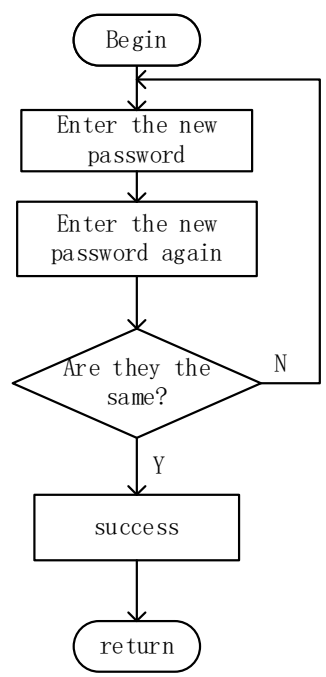

Fig. 5 The changing password subroutine flow chart

\section{The system simulation}

The test results are that if the pressure difference value measured by the pressure detection module is beyond the threshold ,the LED light used to simulate the intelligent anti-theft alarm system will flicker. If the password-input key is pressed the LCD12864 will be on and will show the corresponding interfaces. If the wrong password is entered three times in succession, the LED light used to simulate the intelligent anti-theft alarm system will flicker. The start interface of the display is shown in Fig. 6, the password-input interface of the display is shown in Fig. 7, the option interface of the display is shown in the Fig. 8, if the key 1 is pressed the microcontroller will send control signal to the electric bicycle controller, which will automatically lock the motor and the electric bicycle will be locked. If the key 2 is pressed the microcontroller will send control signal to the electric bicycle controller, which will automatically unlock the motor and the electric bicycle will be unlocked. If the key 3 is pressed the display will show”Please Enter New Password:". After the new 6-bit password is all entered the display will show"Please Enter Again”.When the enter is completed it will show "Change Password Successfully". 


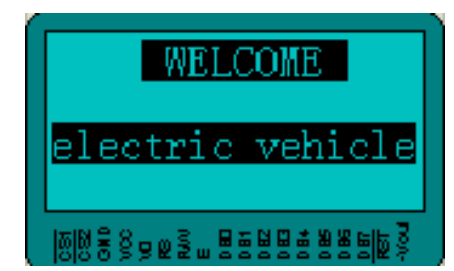

Fig. 6 The start interface of the display

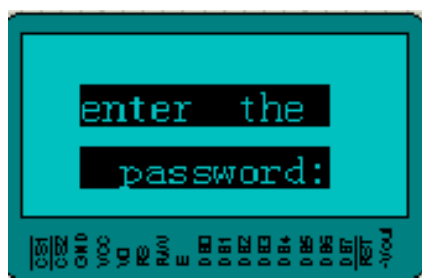

Fig. 7 The password-input interface of the display

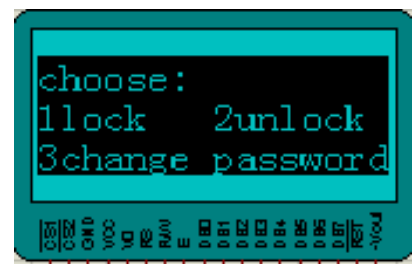

Fig. 8 The option interface of the display

\section{Conclusion}

This paper designed the hardware and software of the intelligent anti-theft system for electric bicycles based on the microcontroller, which can not only much more conveniently and safely lock or unlock the electric bicycles,but also can timely monitor the battery. Simulation results show that the system with low cost and high stability is practical and easy to operate,which can meet the requirements in the aspect of electric bicycles’ anti-theft.

\section{References}

[1] Yang Binfeng,Wang Qiong,Wang Xiaokan.Design and Realization of Multifunctional and Intelligent Protection Instrument for Electric Bicycle[J]. IEEE International Conference on Computer Science and Information Technology, 2010,190-193.

[2] Stephen Prata. C Primer Plus[M]. Peking:Posts \& Telecom Press,2005.

[3] Peter Van Der Linden. Expert C Programming[M]. Peking:Posts \& Telecom Press,2013.

[4] Fu Jiacai chief.The Electronics Engineering Practice Technique[M]. Peking:Chemical Industry Publishing House, 2002.

[5] Zhang Xiaodong,Wu Youcang.The Electrician Practical Electronics Design[M]. Peking:Defence Industry Publishing House,2006.

[6] Wang Junfeng,Meng Qi.Modern Sensor Application Technique[M].Peking:Machine Industrial Publishing House,2006.

[7] Wang Luyan,Chen Hang,Ye Shuming.A Multi-parameter Signal Monitoring System Based on Cortex-M3[J].IEEE Workshop on Electronics,Computer and Application,2014,870-873.

[8] Zhang Haiyan.Design of New Electric Bicycles’ Anti-Theft System[D].Zhengzhou: Zhengzhou University, 2009.

[9] Guo Haiying.Design of Electronic Security Password Lock Based on The microcontroller[J].Modern Electronic Technology, 2005, 28 (13): 95-97. 